\title{
Numerical Solution of Nonlinear Fredholm-Volterra Integtral Equations via Piecewise Constant Function by Collocation Method
}

\author{
Ahmad Shahsavaran \\ Faculty of Science, Islamic Azad University, Borujerd Branch, Borujerd, Iran \\ E-mail: a.shahshavaran@iaub.ac.ir \\ Received March 26, 2011; revised April 17, 2011; accepted May 10, 2011
}

\begin{abstract}
In this work, we present a computational method for solving nonlinear Fredholm-Volterra integral equations of the second kind which is based on replacement of the unknown function by truncated series of well known Block-Pulse functions (BPfs) expansion. Error analysis is worked out that shows efficiency of the method. Finally, we also give some numerical examples.
\end{abstract}

Keywords: Nonlinear Fredholm-Volterra Integral Equation, Block-Pulse Function, Error Analysis, Collocation Points

\section{Introduction}

The integral equation method is widely used for solving many problems in mathematical physics and engineering. This article proposes a computational method for solving nonlinear Fredholm-Volterra integral equations. Several numerical methods for approximating the solution of linear and nonlinear integral equations and specially FredholmVolterra integral equations are known [1-10]. Also, BlockPulse functions are studied by many authors and applied for solving different problems. In presented paper, by using vector forms of BPfs, the main problem can be easily reduced to a nonlinear system of algebraic equations which can be solved by Newton's iterative method.

\section{Review of Some Related Papers}

Some computational methods for approximating the solution of linear and nonlinear integral equations are known. The classical method of successive approximation for Fredholm-Hammerstein integral equations was introduced in [3]. Brunner in [4] applied a collocation type method and Ordokhani in [8] applied rationalized Haar function to nonlinear Volterra-Fredholm-Hammerstein integral equations. A variation of the Nystrom method was presented in [5]. A collocation type method was developed in [6]. The asymptotic error expansion of a collocation type method for volterra-Hammerstein in- tegral equations has been considered in [7]. Yousefi in [9] applied Legendre wavelets to a special type of nonlinear Volterra-Fredholm integral equations of the form.

$$
\begin{gathered}
u(t)=f(t)+\lambda_{1} \int_{0}^{t} K_{1}(t, x) F(u(x)) \mathrm{d} x+\lambda_{2} \\
\int_{0}^{1} K_{2}(t, x) G(u(x)) \mathrm{d} x, 0 \leq x, t \leq 1,
\end{gathered}
$$

where $f(t)$, and $K_{1}(t, x)$ and $K_{2}(t, x)$ are assumed to be in $L^{2}(R)$ on the interval $0 \leq x, t \leq 1$. Yalcinbas in [10] used Taylor polynomials for solving Equation (1) with $F(u)=u^{p}$ and $G(u)=u^{q}$. Orthogonal functions and polynomials receive attention in dealing with various problems that one of those in integral equation. The main characteristic of using orthogonal basis is that it reduces these problems to solving a system of nonlinear algebraic equations. The aim of this work is to present a numerical method for approximating the solution of nonlinear Fredholm-Volterra integral equation of the form:

$$
\begin{gathered}
u(t)=f(t)+\lambda_{1} \int_{0}^{t} K_{1}(t, x)(u(x))^{m} \mathrm{~d} x+\lambda_{2} \\
\int_{0}^{1} K_{2}(t, x)(u(x))^{n} \mathrm{~d} x, 0 \leq x, t \leq 1,
\end{gathered}
$$

where $m$ and $n$ are nonnegative integers and $\lambda_{1}$ and $\lambda_{2}$ are constants. For this purpose we define a k-set of BPfs as

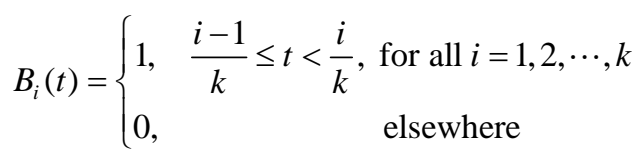


The functions $B_{r}(t)$ are disjoint and orthogonal. That is,

$$
\begin{aligned}
& B_{j}(t) B_{i}(t)=\left\{\begin{array}{cc}
0, & i \neq j \\
B_{i}(t), & i=j
\end{array}\right. \\
& <B_{i}(t) B_{j}(t)>=\left\{\begin{array}{cc}
0, & i \neq j \\
\frac{1}{k}, & i=j
\end{array}\right.
\end{aligned}
$$

A function $u(t)$ defined over the interval $[0,1)$ may be expanded as:

$$
u(t)=\sum_{i=1}^{\infty} u_{i} B_{i}(t) .
$$

In practice, only $k$-term of (6) are considered, where $\mathrm{k}$ is a power of 2 , that is,

$$
u(t) \cong u_{k}(t)=\sum_{i=1}^{k} u_{i} B_{i}(t),
$$

with matrix from:

$$
u(t) \cong u_{k}(t)=u^{t} B(t),
$$

where, $\mathbf{u}=\left[u_{1}, u_{2}, \cdots, u_{k}\right]^{t}$ and

$$
\mathbf{B}(t)=\left[B_{1}(t), B_{2}(t), \cdots, B_{k}(t)\right]^{t} .
$$

In a similar manner, $[u(t)]^{m}$ can be approximated in term of BPfs

$$
[u(t)]^{m} \cong \tilde{\mathbf{u}}^{t} \mathbf{B}(t),
$$

that we need to calculate vector $\tilde{u}$ whose elements are nonlinear combination of the elements of the vector $\mathbf{u}$ For this purpose, we can write

$$
u(t)=\mathbf{u}^{t} \mathbf{B}(t) \text { and }[u(t)]^{m}=\tilde{\mathbf{u}}^{t} \mathbf{B}(t) .
$$

So,

$$
\tilde{\mathbf{u}}^{t} \mathbf{B}(t)=\left[\mathbf{u}^{t} \mathbf{B}(t)\right]^{m}
$$

now using (4) leads to

$$
\mathbf{B}(t) \mathbf{B}^{t}(t)=\left(\begin{array}{cccc}
B_{1}(t) & & & 0 \\
& B_{2}(t) & & \\
& & \ddots & \\
0 & & & B_{k}(t)
\end{array}\right)
$$

also from (3) we get

$0 \leq t<\frac{1}{k}$ implies that $B_{1}(t)=1$ and $B_{i}(t)=0$ for $i=2, \cdots, k$.

$\frac{1}{k} \leq t<\frac{2}{k}$ implies that $B_{2}(t)=1$ and $B_{i}(t)=0$ for $i=1, \cdots, k$ and $i \neq 2$.

$\frac{k-1}{k} \leq t<1$ implies that $B_{k}(t)=1$ and $B_{i}(t)=0$ for $i=1, \cdots, k-1$. Therefore, simply we obtain

$$
\int_{0}^{1} \mathbf{B}(t) \mathbf{B}^{t}(t) \mathrm{d} t=\frac{1}{k} \mathbf{I},
$$

where, I is the identity matrix of order k. By incorporating these results we have

$$
\tilde{\mathbf{u}}^{t}=\tilde{\mathbf{u}}^{t} \mathbf{I}=k \int_{0}^{1} \tilde{\mathbf{u}}^{t} \mathbf{B}(t) \mathbf{B}^{t}(t) \mathrm{d} t=k \int_{0}^{1}\left[\mathbf{u}^{t} \mathbf{B}(\mathrm{t})\right]^{m} \mathbf{B}^{t}(t) \mathrm{d} t .
$$

Hence,

$$
\begin{aligned}
\tilde{\mathbf{u}}^{t} & =k \int_{0}^{1}\left[\mathbf{u}^{t} \mathbf{B}(t)\right]^{m} \mathbf{B}^{t}(t) \mathrm{d} t \\
& =k \sum_{i=1}^{k} \int_{\frac{i-1}{k}}^{\frac{i}{k}}\left[\mathbf{u}^{t} \mathbf{B}(t)\right]^{m} \mathbf{B}^{t}(t) \mathrm{d} t \\
& =k \sum_{i=1}^{k} \int_{\frac{i-1}{k}}^{\frac{i}{k}}\left[\mathbf{u}^{t} \mathbf{B}(t)\right]^{m-1} \mathbf{u}^{t}\left[\mathbf{B}(t) \mathbf{B}^{t}(t)\right] \mathrm{d} t
\end{aligned}
$$

So using (11) leads to

$$
\tilde{\mathbf{u}}^{t}=k \int_{0}^{\frac{1}{k}}\left(\left[u_{1}, u_{2}, \ldots, u_{k}\right]\left(\begin{array}{c}
1 \\
0 \\
\ddots \\
0
\end{array}\right)\right)^{m-1} .
$$

$$
\begin{aligned}
& {\left[u_{1}, u_{2}, \cdots, u_{k}\right]\left(\begin{array}{ccccc}
1 & & & & 0 \\
& 0 & & & \\
& & 0 & & \\
& & \ddots & \\
0 & & & 0
\end{array}\right) \mathrm{d} t} \\
& +k \int_{\frac{1}{k}}^{\frac{2}{k}}\left(\left[u_{1}, u_{2}, \cdots, u_{k}\right]\left(\begin{array}{l}
0 \\
1 \\
0 \\
\ddots \\
0
\end{array}\right)\right)^{m-1}
\end{aligned}
$$

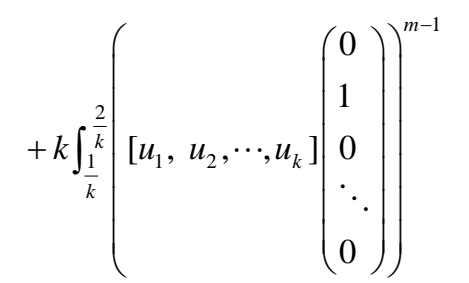

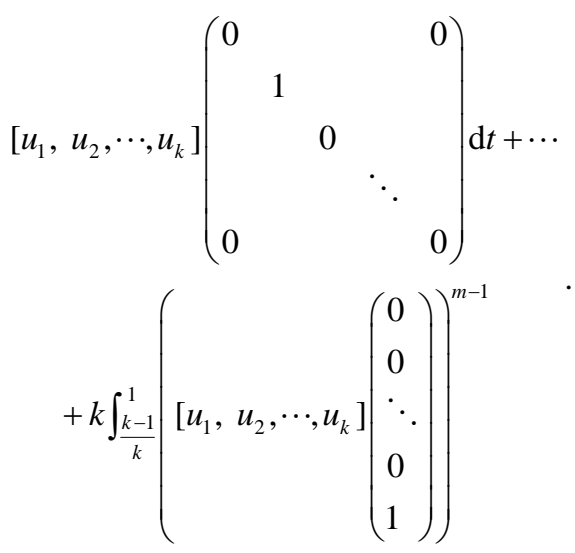

$$
\left[u_{1}, u_{2}, \cdots, u_{k}\right]\left(\begin{array}{ccccc}
0 & & & & 0 \\
& 0 & & & \\
& & \ddots & & \\
& & & 0 & \\
0 & & & & 1
\end{array}\right) \mathrm{d} t
$$




$$
\begin{aligned}
= & k \int_{0}^{\frac{1}{k}} u_{1}^{m-1}\left[u_{1} 0, \cdots, 0\right] \mathrm{d} t+k \int_{\frac{1}{k}}^{\frac{2}{k}} u_{2}^{m-1}\left[0, u_{2}, \cdots, 0\right] \mathrm{d} t+\cdots \\
& +k \int_{\frac{k-1}{k}}^{1} u_{m}^{m-1}\left[0, \cdots, 0, u_{m}\right] \mathrm{d} t=\left[u_{1}^{m}, u_{2}^{m}, \cdots, u_{m}^{m}\right]
\end{aligned}
$$

Now for evaluating the integral $\int_{0}^{t} \mathbf{B}(t) \mathbf{B}^{t}(t) \mathrm{d} t$ at the collocation points

$$
t_{j}=\frac{j-\frac{1}{2}}{k}, j=1,2, \cdots, k,
$$

we may proceed as follows

$$
\begin{aligned}
& \int_{0}^{t_{j}} \mathbf{B}(t) \mathbf{B}^{t}(t) d t=\int_{0}^{\frac{j-1 / 2}{k}} \mathbf{B}(t) \mathbf{B}^{t}(t) \mathrm{d} t \int_{0}^{\frac{1}{k}} \mathbf{B}(t) \mathbf{B}^{t}(t) \mathrm{d} t+ \\
& \int_{\frac{1}{k}}^{\frac{2}{k}} \mathbf{B}(t) \mathbf{B}^{t}(t) \mathrm{d} t+\cdots \\
& +\int_{\frac{j-2}{k}}^{\frac{j-1}{k}} \mathbf{B}(t) \mathbf{B}^{t}(t) \mathrm{d} t+\int_{\frac{j-1}{k}}^{\frac{j-1 / 2}{k}} \mathbf{B}(t) \mathbf{B}^{t}(t) \mathrm{d} t \\
& =\left(\begin{array}{cccc}
\int_{0}^{\frac{1}{k}} 1 \mathrm{~d} t & & & 0 \\
& 0 & & \\
& & \ddots & \\
0 & & & 0
\end{array}\right)+\left(\begin{array}{cccc}
0 & & & 0 \\
& \int_{\frac{1}{k}}^{\frac{2}{k}} 1 \mathrm{~d} t & & \\
& 0 & \ddots & \\
0 & & & 0
\end{array}\right)+\cdots \\
& +\left(\begin{array}{ccccc}
0 & & & & 0 \\
& \ddots & & & \\
& & 0 & & \\
& & \int_{\frac{j-2}{k}}^{\frac{j-1}{k}} 1 \mathrm{~d} t & & \\
& & 0 & & \\
& & & \ddots & \\
0 & & & & 0
\end{array}\right) \\
& +\left(\begin{array}{ccccc}
0 & & & & 0 \\
& \ddots & & & \\
& & 0 & & \\
& & \int_{\frac{j-1}{k}}^{\frac{j-1 / 2}{k}} 1 \mathrm{~d} t & & \\
& & 0 & & \\
& & & \ddots & \\
0 & & & & 0
\end{array}\right)=\frac{1}{k} \mathbf{D}^{j}
\end{aligned}
$$

where,

$$
\mathbf{D}^{j}=\operatorname{Diag}\left[1,1, \cdots, \frac{1}{2}, 0, \cdots, 0\right]_{k \times k},
$$

in fact, the diagonal matrix $\mathbf{D}^{j}, j=1,2, \cdots, k$ is defined as follows :

$$
\mathbf{D}_{m n}^{j}= \begin{cases}1, & m=n=1,2, \cdots j-1, \\ \frac{1}{2}, & m=n=j, \\ 0, & m=n=j+1, \cdots, k .\end{cases}
$$

Also, $K(x, t) \in L^{2}[0,1)^{2}$ may be approximated as:

$$
K(x, t) \cong \sum_{i=1}^{k} \sum_{j=1}^{k} K_{i j} B_{i}(x) B_{j}(t),
$$

or in matrix form

$$
K(x, t) \cong \mathbf{B}^{t}(x) \mathbf{K B}(t),
$$

where $\mathbf{K}=\left[K_{i j}\right]_{1 \leq i, j \leq k}$ and $K_{i j}=k^{2} \int_{0}^{1} \int_{0}^{1} K(x, t) B_{i}(x) B_{j}$ $(t) \mathrm{d} x \mathrm{~d} t$.

\section{Solution of the Nonlinear Fredholm- Volterra Integral Equations}

In order to use BPfs for solving nonlinear FredholmVoterra integral equations given in Equation (2), we first approximate the $u(t), f(t),(u(x))^{m},(u(x))^{n}, K_{1}(t$, $x)$ and $K_{2}(t, x)$ with respect to BPfs

$$
\begin{gathered}
u(t) \cong \mathbf{B}^{t}(t) \mathbf{u} \\
f(t) \cong \mathbf{B}^{t}(t) \mathbf{f} \\
(u(x))^{m} \cong \tilde{\mathbf{u}}_{1}^{t} \mathbf{B}(x) \\
(u(x))^{n} \cong \tilde{\mathbf{u}}_{2}^{t} \mathbf{B}(x) \\
K_{1}(t, x) \cong \mathbf{B}^{t}(t) \mathbf{K}_{1} \mathbf{B}(x) \\
K_{2}(t, x) \cong \mathbf{B}^{t}(t) \mathbf{K}_{2} \mathbf{B}(x)
\end{gathered}
$$

where k-vectors $\mathbf{u}, \mathbf{f}, \tilde{\mathbf{u}}_{1}, \tilde{\mathbf{u}}_{2}$, and $k \times k$ matrices $\mathbf{K}_{1}$ and $\mathbf{K}_{2}$ are BPfs coefficients of $u(t), f(t)$, $(u(x))^{m},(u(x))^{n}, K_{1}(t, x)$ and $K_{2}(t, x)$ respectively. For solving Equation (2), we substitute (15-20) into (2), therefore

$$
\begin{aligned}
\mathbf{B}^{t}(t) \mathbf{u}= & \mathbf{B}^{t}(t) \mathbf{f}+\lambda_{1} \mathbf{B}^{t}(t) \mathbf{K}_{1} \int_{0}^{t} \mathbf{B}(x) \mathbf{B}^{t}(x) \mathrm{d} x \tilde{\mathbf{u}}_{1}, \\
& +\lambda_{2} \mathbf{B}^{t}(t) \mathbf{K}_{2} \int_{0}^{1} \mathbf{B}(x) \mathbf{B}^{t}(x) \mathrm{d} x \tilde{\mathbf{u}}_{2},
\end{aligned}
$$

We now collocate Equation (21) at $k$ points $t_{i}$, $j=1,2, \cdots, k$ defined by (12) as

$$
\begin{aligned}
\mathbf{B}^{t}\left(t_{j}\right) \mathbf{u}= & \mathbf{B}^{t}\left(t_{j}\right) \mathbf{f}+\lambda_{1} \mathbf{B}^{t}\left(t_{j}\right) \mathbf{K}_{1} \int_{0}^{t_{j}} \mathbf{B}(x) \mathbf{B}^{t}(x) \mathrm{d} x \tilde{\mathbf{u}}_{1} \\
& +\lambda_{2} \mathbf{B}^{t}\left(t_{j}\right) \mathbf{K}_{2} \int_{0}^{1} \mathbf{B}(x) \mathbf{B}^{t}(x) \mathrm{d} x \tilde{\mathbf{u}}_{2}
\end{aligned}
$$

by using (10) and (13) and the fact that $\mathbf{B}\left(t_{j}\right)=\mathbf{e}_{j}$ where, $\mathbf{e}_{j}$ is the $j$-th column of the identity matrix of order k, Equation (22) may then be restated as

$$
u_{j}=f_{i}+\frac{\lambda_{1}}{k} \mathbf{e}_{j}^{t} \mathbf{K}_{1} \mathbf{D}^{j} \tilde{\mathbf{u}}_{1}+\frac{\lambda_{2}}{k} \mathbf{e}_{j}^{t} \mathbf{K}_{2} \tilde{\mathbf{u}}_{2}, \quad j=1,2, \cdots, k \text {. }
$$


Table 1.

\begin{tabular}{|c|c|c|c|c|c|}
\hline$t$ & Exact & Approximate for & $k=8$ & Approximate for & $k=16$ \\
\hline 0.1 & $\begin{array}{l}-1.99 \\
\end{array}$ & -1.9847 & & -1.9876 & \\
\hline 0.2 & -1.96 & -1.9505 & & -1.9532 & \\
\hline 0.3 & -1.91 & -1.8857 & & -1.8905 & \\
\hline 0.4 & -1.84 & -1.7905 & & -1.8122 & \\
\hline 0.5 & -1.75 & -1.7650 & & -1.7666 & \\
\hline 0.6 & -1.64 & -1.6650 & & -1.6589 & \\
\hline 0.7 & -1.51 & -1.5091 & & -1.5080 & \\
\hline 0.8 & -1.36 & -1.3205 & & -1.3342 & \\
\hline 0.9 & -1.19 & -1.1103 & & -1.1297 & \\
\hline
\end{tabular}

Table 2.

\begin{tabular}{cccc}
\hline$t$ & Exact & Approximate for $k=8$ & Approximate for $k=16$ \\
\hline 0.1 & 0.0998 & 0.0625 & 0.0936 \\
0.2 & 0.1986 & 0.1866 & 0.2070 \\
0.3 & 0.2955 & 0.3078 & 0.2776 \\
0.4 & 0.3894 & 0.4242 & 0.3952 \\
0.5 & 0.4794 & 0.5139 & 0.5067 \\
0.6 & 0.5646 & 0.5339 & 0.5596 \\
0.7 & 0.6442 & 0.6353 & 0.6514 \\
0.8 & 0.7173 & 0.7268 & 0.7243 \\
0.9 & 0.7833 & 0.8069 & 0.7874 \\
\hline
\end{tabular}

be solved for the elements $\tilde{\mathbf{u}}_{1}$ using Newton's iterative method.

\section{Error in BPfs Approximation}

Theorem. If a differentiable function $u(t)$ with bounded first derivative on $(0,1)$ is represented in a series of $\mathrm{BPf}$ over subinterval $\left[\frac{i-1}{k}, \frac{i}{k}\right)$, we have $\|e(t)\|=O\left(\frac{1}{k}\right)$, where $e(t)=u_{k}(t)-u(t)$.

Proof. See [1].

\section{Illustrative Examples}

Consider the following nonlinear volterra-Fredholm integral equations.

\section{Example 1.}

$$
\begin{aligned}
u(t)= & \frac{-1}{30} t^{6}+\frac{1}{3} t^{4}-t^{2}+\frac{5}{3} t-\frac{5}{4}+ \\
& \int_{0}^{t}(t-x)[u(x)]^{2} d x+\int_{0}^{1}(t+x)[u(x)] d x, \\
& 0 \leq t, \quad x \leq 1 .
\end{aligned}
$$

We applied the method presented in this paper for solving Equation (2) with $k=8$ and $k=16$.

The computational results together with the exact solution $u(t)=t^{2}-2$ are given in Table 1 .

\section{Example 2.}

$$
u(t)=\sin t+\frac{1}{8} \sin 2 t-\frac{1}{4} t+\int_{0}^{t} \frac{1}{2}[u(x)]^{2} \mathrm{~d} x, 0 \leq t, x \leq 1 .
$$

The computational results with $k=8$ and $k=16$ together with the exact solution $u(t)=\sin t$ are given in Table 2.

\section{Conclusions}

The aim of present work is to apply a method for solving the nonlinear Volterra-Fredholm integral equations. The properties of the Block Pulse functions together with the collocation method are used to reduce the problem to the solution of nonlinear algebraic equations. Example 1 is solved in [2] using Chebyshev expansion method (Cem), comparing the results shows Cem is more accurate than BPfs method But, it seems the number of calculations of BPfs method is lower. Also, the benefits of this method are low cost of setting up the equations due to properties of BPfs mentioned in Section 2. In addition, the nonlinear system of algebraic equations is sparse. Finally, this method can be easily extended and applied to nonlinear Volterra-Fredholm integral equations of the form Equation (1). Illustrative examples are included to demonstrate the validity and applicability of the technique.

\section{References}

[1] A. Shahsavaran, E. Babolian, "Numerical Implementation of an Expansion Method for Linear Volterra Integral Equations of the Second Kind with Weakly Singular Kernels,” International Journal of Applied Mathematics, Vol. 3, No. 1, 2011, pp. 1-8.

[2] E. Babolian, F. Fattahzadeh and E. G. Raboky, "A Chebyshev Approximation for Solving Nonlinear Integral Equations of Hammerstein Type,” Applied Mathematics and Computation Vol. 189, No. 1, 2007, pp. 641-646. doi:10.1016/j.amc.2006.11.181

[3] F. G. Tricomi, "Integral equations”, Dover, 1982.

[4] H. Brunner, "Implicity Linear Collocation Method for Nonlinear Volterra Equations," Applied Numerical Mathematics, Vol. 9, No. 3-5, 1982, pp. 235-247. doi:10.1016/0168-9274(92)90018-9

[5] L. J. Lardy, “A Variation of Nysrtom's Method for Hammerstein Integral Equations,” Journal of Integral Equations, Vol. 3, No. 1, 1982, pp. 123-129.

[6] S. Kumar, I. H. Sloan, “A New Collocation-Type Method for Hammerstein Integral Equations,” Journal of Computational Mathematics, Vol. 48, No. 178, 1987, 585-593.

[7] H. Guoqiang, "Asymptotic Error Expansion Variation of A Collocation Method for Volterra-Hammerstein equations,” Applied Numerical Mathematics, Vol. 13, No. 5, 1993, pp. 357-369. doi:10.1016/0168-9274(93)90094-8

[8] Y. Ordokhani, "Solution of Nonlinear Volterra-Fred- 
holm-Hammerstein Integral Equations Via Rationalized Haar Functions," Applied Mathematics and Computation, Vol. 180, No. 2, 2006, pp. 436-443. doi:10.1016/j.amc.2005.12.034

[9] S. Yousefi and M. Razzaghi, "Legendre Wavelet Method for the Nonlinear Volterra-Fredholm Integral Equations," Mathematics and Computers in Simulation, Vol. 70, No.
1, 2005, pp. 1-8. doi:10.1016/j.matcom.2005.02.035

[10] S. Yashilbas, "Taylor Polynomial Solution of Nonlinear Volterra-Fredholm Integral Equations,” Applied Mathematics and Computation, Vol. 127 No. 2-3, 2002, pp. 195-200. doi:10.1016/S0096-3003(00)00165-X 